\title{
SRF-FOXO1 and SRF-NCOA1 fusion genes delineate a distinctive subset of well differentiated rhabdomyosarcoma
}

Running Title: SRF-fused Rhabdomyosarcoma

Marie Karanian ${ }^{1,2}$, Daniel Pissaloux ${ }^{1,2}$, Anne Gomez-Brouchet ${ }^{3}$, Carole Chevenet ${ }^{4}$, François Le Loarer ${ }^{5}$, C Fernandez ${ }^{6}$, Veronique Minard ${ }^{7}$, Nadege Corradini ${ }^{8}$, MariePierre Castex ${ }^{9}$, Adeline Duc-Gallet ${ }^{2}$, Jean-Yves Blay ${ }^{2,11}$ and Franck Tirode ${ }^{2}$.

1 Department of Biopathology, Centre Leon Berard, Lyon, France.

2 Lyon University, Claude Bernard University Lyon 1, INSERM 1052, CNRS 5286, Cancer Research Center of Lyon CRCL, Lyon, France.

${ }^{3}$ Department of Pathology, IUCT-oncopole, University Hospital of Toulouse, Toulouse, France.

${ }^{4}$ Department of Pathology, University Hospital Estaing, Clermont-Ferrand, France.

${ }^{5}$ Department of Pathology, Institut Bergonie, Bordeaux, France.

${ }^{6}$ Department of Pathology, Saint-Denis University Hospital Felix Guyon, Réunion, France.

7 Department of Pediatric and Adolescent Oncology, Institut Gustave Roussy (GR), Villejuif, France

${ }^{8}$ Department of Pediatric and Adolescent Oncology, IHOP, Centre Leon Berard, Lyon, France.

${ }^{9}$ Department of Pediatric and Adolescent Unity Oncology, Toulouse University Hospital, Toulouse, France.

${ }^{11}$ Department of Medical Oncology, Centre Leon Berard, Lyon, France

\section{Corresponding author:}

Marie Karanian, Pathology department, Centre Léon Bérard,28 Rue Laënnec, 69373 Lyon Cedex 08, France. E-mail: marie.karanian@lyon.unicancer.fr; Phone : +33478782920

\section{Acknowledgment}

This study was supported by the Institut National du Cancer and the French Health Ministry (Direction Générale de l'Offre de Soins), grant INCa-DGOS_13219 (iMAPS project).

\section{Conflict of interest:}

The authors have disclosed that they have no significant relationships with, or financial interest in, any commercial companies pertaining in this study. 


\begin{abstract}
Rhabdomyosarcoma encompasses a heterogeneous collection of tumors in which new groups have recently been identified that improved the WHO classification. While performing RNAsequencing in our routine practice, we identified three cases of welldifferentiated rhabdomyosarcoma harboring new fusion genes. We also analyzed these tumors through array-CGH. Clinically, these tumors were deep paraspinal tumors, occurring in neo-nat and young children. The patients underwent resection and adjuvant therapy. At the time of last follow-up (ranging from 12-108 months) they were alive without disease. Histologically, these tumors consisted of well-differentiated rhabdomyoblastic proliferations with nuclear atypia, infiltrative borders and a specific growth pattern. These tumors harbored new fusion genes involving SRF and either FOXO1 or NCOA1. We compared the expression profiles of these three tumors to the expression data of a series of 33 skeletal muscle tumors including embryonal rhabdomyosarcomas, alveolar rhandomyosarcomas, rhabdomyosarcomas with VGLL2 fusions, rhabdomyosarcomas with myoD1 mutation, EWSR1/FUS-TFCP2 epithelioid and spindle cell rhabdomyosarcomas of the bone and rhabdomyomas with PTCH1 loss. According to clustering analyses, the three SRF-fused tumors formed a distinct group with a specific expression profile different from that of the other types of skeletal muscle tumors. Array CGH showed a recurrent gain of chromosome 11. These three tumors define a new group of rhabdomyosarcoma associated with a fusion of the SRF gene. FOXO1 rearrangements, usually used to confirm the diagnosis of alveolar rhabdomyosarcoma and identify poor outcome rhabdomyosarcomas, were identified in a non-alveolar rhabdomyosarcoma for the first time.
\end{abstract}

Key words: Rhabdomyosarcoma, SRF, FOXO1, NCOA1, sarcoma, RNAsequencing, Array-comparative genomic hybridization, Fluorescence in situ hybridization (FISH). 


\section{Introduction}

Rhabdomyosarcoma (RMS) is the most frequent soft tissue sarcoma in children. According to the latest WHO classification this sarcoma is sub-classified into 4 groups: embryonal RMS, alveolar RMS, pleomorphic RMS and spindle cell/sclerosing RMS(1). Alveolar RMS is a well-defined tumor, characterized by a round cell proliferation pattern and recurrent chromosomal translocations which are used in routine practice as diagnostic markers. Clinically, alveolar RMS occurs in older children and follows an aggressive course. The embryonal RMS type is not associated with a specific molecular abnormality and can show different morphological features and its diagnosis is often supported by the lack of translocation. Clinically, embryonal RMS is commonly diagnosed in younger children and has a better prognosis. These two groups, representing the majority of pediatric cases, are now divided into FOXO1 fusionpositive and FOXO1 fusion-negative tumors. Spindle cell/sclerosing RMS is a heterogeneous group, likely incompassing different types of RMS with different morphological and clinical features. Pleomorphic RMS is a sarcoma with a complex genetic profile occurring predominantly in adults and associated microscopically with a pleomorphic cell proliferation. Recently, several studies have described two kinds of RMSs initially included in the spindle cell/sclerosing RMS: RMSs with rearrangement of the VGLL2 or TEAD1 genes (2-4) and RMSs with a mutation in the MYOD1 gene (5-8). RMSs characterized by a gene fusion involving VGLL2 or TEAD1 with NCOA2 or CITED2 arise during the neonatal period and seem to be less aggressive than classical RMS(3). RMSs with MYOD1 mutations occur in children and in adults and have a poor outcome(8). A novel type of translocation-related RMS was also recently reported: intra osseous RMS with a EWSR1- or FUS-TFCP2 $(4,9)$ and MEIS1-NCOA2 fusion gene(10). Finally, a new group of borderline skeletal muscle tumors termed "histiocyte-rich rhabdomyoblastic tumor" was identified, the authors adding a new category of intermediate malignancy to the group of skeletal muscle tumors(11).

Defining a tumor entity relies on an ensemble of specific clinical, morphological and molecular features. Herein we report three cases of well-differentiated RMS, forming a homogeneous group, both clinically and histologically. While investigating these tumors through $\mathrm{CGH}$ array and RNA-sequencing we identified new fusion genes joining SRF with FOXO1 or NCOA1. These three samples also shared a specific transcriptional profile. Altogether, these tumors were different from classical RMS or usual rhabdomyomas, thus meeting all of the criteria to be considered as a distinct subset of skeletal muscle tumors.

\section{Material and Methods}

\section{Patient samples}

The three cases were sent to the pathology department of the Centre Leon Berard for a second opinion. These samples were acquired with informed consent from patients and ethical approval from the research committee board of the Centre Leon Berard. Patient samples were anonymized and used in accordance with the principles expressed in the Declaration of Helsinki. Clinical findings were retrieved from medical files.

\section{Histology and Immunohistochemistry}

Histological examination was performed on $4 \mu \mathrm{m}$-thick sections cut from formalin-fixedparaffin-embedded (FFPE) tissue and stained with hematoxylin and eosin. The 
following primary antibodies were used: anti-Ki-67 (1/100; rabbit monoclonal, clone SP6, Thermo Fischer Scientific USA), Desmin (1/80; mouse monoclonal, clone D33, Agilent, Courtaboeuf, France), Myogenin (1/1; mouse monoclonal, clone F5D, Agilent), Myod1 (1/1, mouse EP212, Roche diagnostics). Immunostaining was performed on a VentanaBenchmark XT automatic stainer (Ventana, Tuscon, AZ) and revealed with the ultraview Universal Dab Detection kit.

\section{RNA sequencing}

Total RNA was extracted from FFPE tissue sections using Trizol reagent (Thermo Fisher scientific, Courtaboeuf, France) and phenol/chloroform. RNase-free DNase set (Qiagen, Courtabouef, Farance) was used to remove DNA. The DNAse was eliminated by a new Trizol extraction. All RNAs were quantified by NanoDrop (ThermoFisher Scientific) and quality was controlled (DV200 value cutoff $>30 \%$ ) by TapeStation with Hs RNA Screen Tape (Agilent, Courtaboeuf, France). $100 \mathrm{ng}$ of total RNA were used to prepare the libraries with the TruSeq RNA Access Library Prep Kit (Illumina, San Diego, USA). 14 libraries were pooled at $4 \mathrm{nM}$ with $1 \%$ PhiX. Sequencing was performed (75 cycles paired end) with NextSeq 500/550 High Output V2 kit on an Illumina NextSeq 500 (Illumina) machine. The fusion transcripts were called with STAR-Fusion, FusionMap, FusionCatcher and EricScript and further considered if present in the fusion list of at least two algorithms(12-15). Expression profiles were extracted from fastq files with Kallisto(16) and transformed as log2(TPM+2) prior to quantile normalization using the Limma package v3.32.2 performed in the $R$ environment v3.4.1(17). Only genes with a coding sequence annotation (based on Ensembl GRCh38p5 annotation) and with a maximum expression value above 2 across all samples were further considered. Consensus clustering analysis was performed using Ward's distance and 1000 samplings of $80 \%$ of both genes and samples and t-SNE analysis was done with a perplexity of 4, using the ConsensusClusterPlus v1.46.0 and Rtsne v0.15 R packages, respectively. Supervised analyses were performed using a Welsh t-test and gene set enrichment analysis(18) was done using gsea-3.0 with all of the gene sets of MSigDB v6.2.

\section{FISH analysis}

FISH was performed on $4 \mu$ m-thick sections of formalin-fixed paraffin-embedded tissue, using the Zytolight FISH-Tissue implementation kit (Zyto Vision Z-2028-20, Bremerhaven, Germany) and the NCOA1 Break Appart (Orange/Green) Empire Genomics (Amplitech, NCOA1BA-20-ORGR), SRF Break appart (Orange/Green) Empire Genomics (Amplitech, SRFBA-20-ORGR FOXO1), and ZytoVision ZytoLight SPEC FKHR Dual Color Break Apart Probe (CliniSciences, Z-2139-50as) per manufacturer's instructions. FISH signals were enumerated in at least 50 nonoverlapping intact nuclei. A specimen was considered positive if more than $20 \%$ of nuclei displayed a signal pattern consistent with a rearrangement (split of orange and green signals or single orange signals without a corresponding green signal.

\section{CGH array}

DNA extraction was performed using the QIAamp DNA micro kit (Qiagen \#56304, Hilden, Germany) on macro-dissected FFPE tumor blocks. Fragmentation and labeling were done according to manufacturer's protocol (Agilent Technologies, Courtaboeuf, France), using $1.5 \mu \mathrm{g}$ of genomic DNA. Tumor DNA was labeled in Cy5, and a female reference DNA (Promega \#G1521, Madison, USA) was labeled in Cy3. Labeled samples were then purified using KREApure columns (Agilent Technologies \#5190- 
0418). Labeling efficiency was calculated using a Nanodrop ND2000 Spectrophotometer. Cohybridization was performed on custom oligonucleotide arrays (Agilent Technologies \#G4125A). These arrays are densified for some cancer genes loci, based on the $4 \times 180 \mathrm{~K}$ Agilent SurePrint G3 Human whole-genome design. Slides were washed, dried, and scanned on the Agilent SureScan microarray scanner. Scanned images were processed using the Agilent Feature Extraction software V11.5 and the analysis was carried out using the Agilent Genomic Workbench software V7.0.

\section{Sanger sequencing}

Reverse transcription was performed with the High-Capacity cDNA Reverse Transcription Kit (Applied Biosystems \#4368814, ThermoFisher Scientific, Courtaboeuf, France) on $1 \mu \mathrm{g}$ of RNA prior to PCR amplification (Q5-high Fidelity-PCR kit \#E0555S, New England Biolabs, Evry, France) using the following primers: 5'AGTGCAGGCCATTCAAGT3' (SRF exon 4, forward) and 5'GTGAGCTAATACACTGGTCTTCT3' (NCOA1 exon 13, reverse) for the SRFNCOA1 in frame fusion and 5'AGTGCAGGCCATTCAAGT3' (SRF exon 6, forward) with 5'TGGATGCAGCTCTTCTCCTA3' (FOXO1 exon 2, reverse) for the SRF-FOXO1 fusion. Sanger sequencing was performed by Eurofins Genomics sequencing service (Ebersberg, Germany) using the NCOA1 exon 13, reverse and FOXO1 exon 2, reverse primers.

\section{Results}

\section{Clinical Features}

Clinical features of the three cases are summarized in Table1.

Case 1 was a one-year-old boy with a congenital neck mass. This $6 \mathrm{~cm}$ in diameter tumor was deeply situated in para-spinal muscles. The tumor was removed by incomplete resection and the diagnosis of rhabdomyosarcoma was made on the specimen. The patient underwent a second complete resection and received chemotherapy. Nine years later, he is alive without disease.

Case 2 was a ten-month-old girl presenting a $6.5 \mathrm{~cm}$ mass in the paraspinal muscles of the neck. The first resection was incomplete. She received chemotherapy and underwent a second resection with free margins. Twelve months later, there is no evidence of a recurrent disease.

Case 3 was a three-year-old boy with a $6 \mathrm{~cm}$ mass situated in the para-spinal muscles of the neck. The tumor was removed but the resection was incomplete. He received chemotherapy. Six months later, a local relapse was detected. The patient underwent a resection with free margins and radiotherapy. After 18 months of follow-up, he is alive without disease.

\section{Pathological Features}

The distinctive feature of these three cases was the predominance of well differentiated myoblasts/rhabdomyoblasts which made the diagnosis challenging: for each case the pathologist took into consideration the diagnoses of well differentiated rhabdomyosarcoma and fetal rhabdomyoma. But, given the infiltrative borders and the nuclear atypia, the diagnosis of rhabdomyosarcoma was proposed.

These tumors were characterized by the same growth pattern: at the periphery myoblasts displayed moderate nuclear atypia and formed small bundles in fibrous 
stroma (Figure 1A, 1C, 1E); at the center spindle and rhabdoid myoblasts showing marked atypia formed large areas of different patterns. For case 1, central areas were composed of long intersecting fascicles of a mixture of spindle and rhabdoid cells which showed abundant eosinophilic cytoplasm and atypical large nuclei with dense and granular chromatin (Figure 1B). Neither mitosis nor necrotic area were observed. The center of case 2 showed areas with a predominance of spindle cells, areas with a predominance of rhabdoid cells and areas with a mixture of these 2 kinds of myoblasts (figure1D). These cells showed marked nuclear atypia and several mitoses. These two kinds of myoblasts were present in case 3 but the component of cells with globoid eosinophilic cytoplasm and eccentric round nuclei was predominant (figure 1F). These cells exhibited atypical nuclear features with large nuclei containing prominent nucleoli but no mitosis.

By immunohistochemistry, these 3 tumors expressed desmin (diffuse staining) (Figure $2 \mathrm{~A}-\mathrm{C}$ ), myogenin and myoD1 (heterogeneous staining) (Figure 2D-F). Ki-67 staining (figure 2G-I) showed a variable proliferation rate: $5 \%$ for case 1, 20\% for case 2 and $10 \%$ for case 3 .

\section{Molecular findings}

\section{Fusion gene detection}

RNA-sequencing was performed on all three samples to investigate the presence of fusion genes (table2). A fusion gene involving the $S R F$ gene was detected in all samples: a SRF-NCOA1 in tumor of case 1 and case 3 and a SRF-FOXO1 in case2. The two SRF-NCOA1-positive samples presented a fusion of the exon 2 of SRF (ENST00000265354) to the exon 13 on NCOA1 (ENST00000407230), leading to a frameshift fusion gene. Nevertheless, a second in frame isoform, joining SRF exon 4 to NCOA1 exon 13, was found in case 3 (Figure 3A). The case 2 presented an in-frame SRF-FOXO1 gene (Figure 3B), joining SRF exon 6 (ENST00000265354) to FOXO1 exon 2 (ENST00000379561).

To validate the RNA-sequencing results, we performed a RT-PCR around the fusion points followed by Sanger sequencing. The in-frame isoform of SRF-NCOA1 was confirmed in case 3 but also identified in case 1 while it was not detected by RNAsequencing (Figure $3 A$ and Table 2). The SRF-FOXO1 in frame fusion gene was also confirmed in case 2 (Figure 3B). In both SRF-FOXO1 and SRF-NCOA1 fusion proteins, the MAD box DNA binding domain of SRF was retained as well as the transcriptional activator domains of NCOA1 or FOXO1, therefore creating aberrant transcription factors.

\section{Genomic analyses}

A FISH break-apart assay was performed on the SRF, FOXO1 and NCOA1 loci in order to cross-validate the presence of the rearrangements and to analyze the clonality of the fusion events. A rearrangement of SRF was identified in $80 \%$ to $90 \%$ of cells in all three samples (Figure 4A). Concomitantly, $70 \%$ of cells presented a rearrangement of NCOA1 in case 1 and case 3 and $80 \%$ of the cells from case 2 showed a FOXO1 rearrangement (Figure $4 \mathrm{~B}-\mathrm{C}$ ).

Next we performed $\mathrm{CGH}$ analyses to evaluate whether the samples presented other genomic alterations (Supplementary data Figure). Copy number variation profiles differed amongst the three samples at the exception of a gain of the chromosome 11 that was present in all samples. In the two SRF-NCOA1-positive tumors, CGH analysis 
also revealed a whole chromosome $X$ gain and segmental imbalances on chromosomes 1,2 and 6 (case 1) or a segmental loss on chromosome 16q (case 3). The SRF-FOXO1-positive tumor (case 2) presented a more complex genomic profile with chromosome $3,8,11,17,20$ and $X$ gains, and 6p25.3-21.1 and 13q14.11-q31.3 (FOXO1 locus) focal gains. In both case 1 and case 2, a breakpoint on 6p21 (SRF locus) was detected. None of the three samples presented a loss of the PTCH1 locus.

\section{Gene expression analyses}

We next wondered whether these tumors could also present a specific gene signature. First, we performed unsupervised analyses (consensus hierarchical clustering and tdistributed stochastic neighbour embedding reduction) of their expression profiles together with those of a larger cohort constituted of different types of rhabdomyosarcomas that were sequenced in our practice during molecular routine diagnoses. In addition to our three samples, this cohort included 6 classical embryonal RMS, 8 alveolar RMS, 4 spindle cells RMS with VGLL2/TEAD1-NCOA2/CITED2 rearrangement, 3 spindle cells RMS without gene fusion, 3 RMS with a MYOD1 p.L122R mutation, 2 cases of rhabdomyoma harboring a $\mathrm{PTCH} 1$ loss and 7 EWSR1/FUS-TFCP2 positive epithelioid and spindle cell RMS. As shown in Figures 5 and 6, except for spindle cells RMS, the different types of RMS formed homogeneous transcriptional groups. Moreover, our three well-differentiated RMSs clustered together and formed a group clearly separated from other skeletal muscle tumor types.

Based on this transcriptomic difference, we performed a supervised analysis to compare SRF-fused RMSs with other types of RMS. As expected, upregulated genes involved in muscle differentiation and function were strongly enriched while a down regulation of cell cycle/proliferation pathways was observed (Figure 5B). Of note, genes harbouring a SRF binding site were also up-regulated which is consistent with the presence of the SRF DNA binding site in the fusion gene. Finally, when focusing on the genes specifically expressed in these three samples, we identified the ADAM Metallopeptidase With Thrombospondin Type 1 Motif 8 (ADAMTS8) gene, which has a 30-fold higher expression in SRF-fused RMSs than in any of the other types of RMSs (Figure 5C).

\section{Discussion}

Rhabdomyosarcoma, is a heterogeneous group of tumors and the current classification, which includes 4 different groups of RMS, is insufficient to describe all types of RMSs encountered in routine practice. Over recent years, new types of RMSs have emerged. In this paper we describe a homogeneous type of well-differentiated RMS defined by specific translocations of the SRF (Serum Response Factor) gene. Clinically, these tumors were deeply situated in paraspinal muscles and occurred during the neonatal period to an early childhood. Histologically, case 3 looked different from the two other cases because it was composed of a majority of rhabdoid cells. However, all of these cases were characterized by: (i) infiltrative borders, (ii) welldifferentiated rhabdomyoblastic atypical cells and (iii) a zonal pattern with small nodules at the periphery and large cellular areas at the center. Infiltrative borders and cellular atypia ruled out the diagnosis of fetal rhabdomyoma. This tumor type did not fit the current classification of RMS. This very well-differentiated pattern is unusual in alveolar RMS, and spindle cell/sclerosing RMS. Embryonal RMS is frequently composed of mesenchymal cells in different stages of myogenesis that can contain numerous rhabdomyoblasts, most often mixed with less differentiated cells. Our cases 
could be included in this definition. Nevertheless, the routine RNA-sequencing performed in our institute for molecular diagnosis, exposed new fusion genes, SRFFOXO1 and SRF-NCOA1 and these clonal abnormalities, confirmed by FISH analysis, cast doubt on an embryonal RMS diagnosis. A fusion gene involving SRF and another member of the Steroid Receptor Coactivators (SRCs) NCOA2 was previously described in a spindle cell RMS(2). This case was included in the group of the recently reported spindle cells RMS harboring a VGLL2-NCOA2 fusion but in which no rhabdomyoblastic differentiation was reported (2-4). We therefore compared the histological aspect of our 3 well-differentiated RMSs with the 3 RMSs of the VGLL2fused family. Except for one case associated with the TEAD1-NCOA2 fusion that showed rare scattered cells with a rhabdomyoblastic aspect, RMS with VGLL2 fusions displayed long fascicles of undifferentiated spindle cells with a scanty cytoplasm whereas all cells of our SRF-fused RMSs samples exhibited a clear muscular differentiation with a large eosinophilic cytoplasm. VGLL2-NCOA2-fused spindle cell RMSs consisted of fascicles on a fibrous stroma whereas SRF-fused welldifferentiated RMSs displayed a specific zonal pattern, characterized by bundles at the periphery and long highly cellular fascicles at the center. In our small series, the histological aspect of the SRF-fused RMSs was therefore extremely different from that of the VGLL2-fused RMS. This dichotomy was further supported by gene expression profiling. Given the fact that clustering analyses are reliable tools to distinguish subtypes of sarcoma $(4)(19,20)$, unsupervised clustering analyses clearly discriminated the two groups. Globally, their expression profiles also differed from those of embryonal RMS, alveolar RMS, spindle cell RMS, EWSR1/TFCP2-positive RMS and rhabdomyoma with $\mathrm{PTCH} 1$ loss. We found an overexpression in the three samples of the ADAMTS8 RNA, a protein that was shown to antagonize the MAPK pathway(21). ADAMTS8 was also noted to be silenced by DNA methylation in numerous cancers, but its overexpression was not reported. Therefore, whether ADAMTS8 plays a role in the biology of well-differentiated RMSs is unknown, but it may serve as a specific biomarker for this entity.

The genomic profiles of the well-differentiated RMSs reported herein also highlighted the difference between this group and the other types of RMSs. Indeed, SRF-fused RMSs were characterized by few whole chromosome or large parts of chromosome gains (with a recurrent chromosome 11 gain) and very few losses of heterozygosity. These profiles are different from those usually described in embryonal RMS, harboring numerous gains and losses, and those of alveolar RMS mostly characterized by genomic amplification and translocation(19,20).

Altogether, histological, genomic and transcriptomic examinations argue in favor of the SRF-fused RMS being a new specific subtype of skeletal muscle tumor.

The prognosis of this entity needs to be evaluated on a larger scale, but given the histological aspects described above, we considered them as rhabdomyosarcomas and the patients underwent the treatment indicated for RMS. For cases 2 and 3, the SRF fusion was identified only a few weeks after the histological diagnosis and supported the fact that these RMSs did not match the current classification. The relapse reported in one case, calls for caution in the care of this type of RMSs.

Other fusion genes involving $S R F$ were identified in soft tissue tumors displaying muscle differentiation: SRF-RELA has been reported in atypical myopericytic tumors(9) and SRF-E2F1 in myoepithelial tumors(22). SRF, located on 6p21, encodes a MADS box family transcriptional factor which regulates, through binding to the CArG 
box motif, genes involved in cell growth, migration, cytoskeletal organization, and myogenesis(23). SRF plays a role in cell differentiation in skeletal, smooth and cardiac muscles(24). Mutant mice lacking skeletal muscle SRF expression died during the neonatal period because of muscle hypotrophy, confirming that SRF controls myofiber growth and maturation(25). The predicted chimeric proteins SRF-NCOA1 and SRFFOXO1 retained the CArG box motif.

Rearrangements of NCOA1 have been reported in alveolar RMS cases $(26,27)$ and biphenotypic sinonasal sarcoma $(28,29)$. Interestingly, biphenotypic sinosal sarcomas with the PAX3-NCOA1 fusion gene showed skeletal muscle differentiation(28). NCOA1 (2p23.3), encodes for the SRC1 protein which belongs to the p160 Steroid Receptor Coactivator (SRC) family that includes SRC1, SRC2 and SRC3(30). SRC proteins contain three structural domains: the N-terminal bHLH-PAS domain interacting with several transcription factors, the central region with three LXXLL motifs responsible for interacting with nuclear receptors and the $\mathrm{C}$-terminal transcriptional activation domains (AD1 and AD2). AD1 binds CBP and p300 histone acetyltransferase (HAT) leading to a transcriptional activation. AD2 interacts with CARM1 (coactivator-associated arginine methyltransferase 1) and PRMT1 (protein arginine methyltransferases), both being involved in histone methylation on arginine residues. SRC-1 and SRC-3 contain HAT activity domains(31,32). These C-terminal domains, involved in chromatin remodeling, were retained in the predicted SRF-NCOA1 fusion protein.

FOXO1 rearrangements are implicated in soft tissues sarcomas: PAX3-FOXO1 and PAX7-FOXO1 were described in alveolar RMS(33-36) and PAX3-FOXO1 was identified in biphenotypic sinonasal sarcoma(29). Concerning the RMSs, FOXO1 fusion has been recognized as a biomarker of poor free-event survival and FISH analysis using FOXO1 (13q14.11) probe is now systematically performed when RMS is suspected (25-28). For the first time, we have identified a FOXO1 rearrangement in a non-alveolar RMS. Though, extremely rare, this result questions the use of FOXO1 rearrangement as a diagnostic and prognostic marker independently of the histological type.

FOXO1 belongs to the forkhead box protein $\mathrm{O}$ (FoxO) transcription factor family and plays an important role in skeletal muscle differentiation and fiber type specification(37). This transcriptional factor contains a forkhead domain (encoded by portions of exons 1 and 2) and a transcriptional activation domain (encoded by exon 2)(36). In the SRF-FOXO1 predicted fusion protein, the C-terminal transcriptional activation domain is retained.

Altogether, these 3 well-differentiated RMSs harbored a chromosomal translocation involving genes implicated in the myogenesis process. The two chimeric proteins contain the DNA binding domain of SRF and the transcription activator domain of FOXO1 or NCOA1. The resulting aberrant transcription factors may therefore be responsible for the well differentiated phenotype observed in these tumors. In accordance with this hypothesis, the expression profiles demonstrated a strong enrichment in genes partaking in muscle biology, involving FOXO1 and SRF responsive genes.

In conclusion, we described here a small series of three well-differentiated RMSs harboring two new fusion genes: SRF-NCOA1 and SRF-FOXO1. These cases form a distinct clinical, histological and transcriptional entity. The collection of further analogous cases is now required in order to evaluate the prognosis of this entity. 
Similarly to the other types of recently identified RMSs associated with translocation or mutation, this new group of skeletal muscle tumors should improve the classification of RMS. Furthermore, our study highlights a FOXO1 rearrangement which is currently used as a diagnostic and prognostic marker, in a non-alveolar RMS. This challenges the use of the FOXO1 probe during FISH analysis as a routine diagnostic test for RMS.

\section{Acknowledgments:}

The authors thank Florine Dreux, Elise Malandin, Cyril Py for their technical help. The authors thank Pr Françoise Galateau-Salle, Dr Allen Gibbs and Brigitte Manship for their assistance with the manuscript. This study was supported by the Institut National du Cancer and the Direction Generale de l'offre de soins, project iMAPS (INCaDGOS_13219).

\section{References}

1. Christopher Fletcher, Julia Bridge, Pancras Hogendoorn et al. WHO Classification of tumors of soft tissue and bone 4th edition. Lyon: IARC; 2013.

2. Mosquera JM, Sboner A, Zhang L, et al. Recurrent NCOA2 gene rearrangements in congenital/infantile spindle cell rhabdomyosarcoma. Genes Chromosomes Cancer 2013;52:538--50.

3. Alaggio R, Zhang L, Sung Y-S, et al. A Molecular Study of Pediatric Spindle and Sclerosing Rhabdomyosarcoma: Identification of Novel and Recurrent VGLL2related Fusions in Infantile Cases. Am J Surg Pathol. 2016;40:224--35.

4. Watson S, Perrin V, Guillemot D et al. Transcriptomic definition of molecular subgroups of small round cell sarcomas. J Pathol 2018;245:29--40.

5. Agaram NP, Chen C-L, Zhang L, et al. Recurrent MYOD1 mutations in pediatric and adult sclerosing and spindle cell rhabdomyosarcomas: evidence for a common pathogenesis. Genes Chromosomes Cancer 2014;53:779-87.

6. Kohsaka S, Shukla N, Ameur N, et al. A recurrent neomorphic mutation in MYOD1 defines a clinically aggressive subset of embryonal rhabdomyosarcoma associated with PI3K-AKT pathway mutations. Nat Genet 2014;46:595--600.

7. Rekhi B, Upadhyay P, Ramteke MP, et al. MYOD1 (L122R) mutations are associated with spindle cell and sclerosing rhabdomyosarcomas with aggressive clinical outcomes. Mod Pathol 2016;29:1532--40.

8. Agaram NP, LaQuaglia MP, Alaggio R, et al. MYOD1-mutant spindle cell and sclerosing rhabdomyosarcoma: an aggressive subtype irrespective of age. A reappraisal for molecular classification and risk stratification. Mod Pathol 2019;32:27--36.

9. Le Loarer F, Cleven AHG, Bouvier C, et al. A subset of epithelioid and spindle cell rhabdomyosarcomas is associated with TFCP2 fusions and common ALK upregulation. Mod Pathol 2019; doi: 10.1038/s41379-019-0323-8

10. Agaram NP, Zhang L, Sung Y-S, et al. Expanding the Spectrum of Intraosseous Rhabdomyosarcoma: Correlation Between 2 Distinct Gene Fusions and Phenotype. Am J Surg Pathol 2019;43:695--702.

11. Martinez AP, Fritchie KJ, Weiss SW, A et al. Histiocyte-rich rhabdomyoblastic tumor: rhabdomyosarcoma, rhabdomyoma, or rhabdomyoblastic tumor of uncertain malignant potential? A histologically distinctive rhabdomyoblastic tumor in search of a place in the classification of skeletal muscle neoplasms. Mod Pathol 2019;32:446--457. 
12. Ge H, Liu K, Juan T, Fang F, et al. FusionMap: detecting fusion genes from nextgeneration sequencing data at base-pair resolution. Bioinforma Oxf Engl. 15 2011;27:1922--8.

13. Haas B, Dobin A, Stransky N, et al. STAR-Fusion: Fast and Accurate Fusion Transcript Detection from RNA-Seq. BioRxiv 2017:120295. doi:10.1101/120295.

14. Nicorici DS, Edgren H, Kangaspeska S, et al. FusionCatcher - a tool for finding somatic fusion genes in paired-end RNA-sequencing data. BioRxiv 2014:011650. doi:10.1101/011650.

15. Benelli M, Pescucci C, Marseglia G, et al. Discovering chimeric transcripts in paired-end RNA-seq data by using EricScript. Bioinforma Oxf Engl 2012;28:3232-9.

16. Bray NL, Pimentel H, Melsted $P$, et al. Near-optimal probabilistic RNA-seq quantification. Nat Biotechnol. 2016;34:525--7.

17. Development Core Team. R: A language and environment for statistical computing. R Foundation for Statistical Computing, Vienna, Austria. 2017. Available at: https://www.R-project.org/.

18. Subramanian A, Tamayo P, Mootha VK, et al. Gene set enrichment analysis: a knowledge-based approach for interpreting genome-wide expression profiles. Proc Natl Acad Sci 2005;102:15545--50.

19. Laé M, Ahn EH, Mercado GE, et al. Global gene expression profiling of PAX-FKHR fusion-positive alveolar and PAX-FKHR fusion-negative embryonal rhabdomyosarcomas. J Pathol 2007;212:143--51.

20. Williamson $D$, Missiaglia $E$, de Reyniès $A$, et al. Fusion gene-negative alveolar rhabdomyosarcoma is clinically and molecularly indistinguishable from embryonal rhabdomyosarcoma. J Clin Oncol 2010;28:2151--8.

21. Choi GCG, Li J, Wang Y, Li L, et al. The Metalloprotease ADAMTS8 Displays Antitumor Properties through Antagonizing EGFR-MEK-ERK Signaling and Is Silenced in Carcinomas by CpG Methylation. Mol Cancer Res 2014;12:228--38.

22. Urbini M, Astolfi A, Indio V, Tarantino G, et al. Identification of SRF-E2F1 fusion transcript in EWSR-negative myoepithelioma of the soft tissue. Oncotarget 2017;8:60036--45.

23. Pipes GCT, Creemers EE, Olson EN. The myocardin family of transcriptional coactivators: versatile regulators of cell growth, migration, and myogenesis. Genes Dev. 2006;20:1545--56.

24. Coletti D, Daou N, Hassani M, et al. Serum Response Factor in Muscle Tissues: From Development to Ageing. Eur J Transl Myol 2016;26:6008.

25. Li S, Czubryt MP, McAnally J, et al. Requirement for serum response factor for skeletal muscle growth and maturation revealed by tissue-specific gene deletion in mice. Proc Natl Acad Sci 2005;102:1082--7.

26. Wachtel M, Dettling M, Koscielniak E, et al. Gene expression signatures identify rhabdomyosarcoma subtypes and detect a novel $\mathrm{t}(2 ; 2)(\mathrm{q} 35 ; \mathrm{p} 23)$ translocation fusing PAX3 to NCOA1. Cancer Res. 2004;64:5539--45.

27. Sumegi J, Streblow R, Frayer RW, et al. Recurrent $t(2 ; 2)$ and $t(2 ; 8)$ translocations in rhabdomyosarcoma without the canonical PAX-FOXO1 fuse PAX3 to members of the nuclear receptor transcriptional coactivator family. Genes Chromosomes Cancer 2010;49:224--36.

28. Huang S-C, Ghossein RA, Bishop JA, Z et al. Novel PAX3-NCOA1 Fusions in Biphenotypic Sinonasal Sarcoma With Focal Rhabdomyoblastic Differentiation. Am J Surg Pathol.2016;40:51--9. 
29. Andreasen S, Bishop JA, Hellquist $\mathrm{H}$, et al. Biphenotypic sinonasal sarcoma: demographics, clinicopathological characteristics, molecular features, and prognosis of a recently described entity. Virchows Arch Int J Pathol 2018;473:615-26.

30. Leo C, Chen JD. The SRC family of nuclear receptor coactivators. Gene 2000; 245:1--11.

31. Xu J, Wu R-C, O'Malley BW. Normal and cancer-related functions of the p160 steroid receptor co-activator (SRC) family. Nat Rev Cancer 2009;9:615--30.

32. Xu J, Li Q. Review of the in vivo functions of the p160 steroid receptor coactivator family. Mol Endocrinol Baltim Md 2003; 17:1681--92.

33. Douglass EC, Valentine M, Etcubanas E, al. A specific chromosomal abnormality in rhabdomyosarcoma. Cytogenet Cell Genet. 1987;45:148--55.

34. Turc-Carel C, Lizard-Nacol S, Justrabo E, et al. Consistent chromosomal translocation in alveolar rhabdomyosarcoma. Cancer Genet Cytogenet 1986;19:361--2.

35. Wang-Wuu S, Soukup S, Ballard E, et al. Chromosomal analysis of sixteen human rhabdomyosarcomas. Cancer Res 1988;48:983--7.

36. Barr FG. Gene fusions involving PAX and FOX family members in alveolar rhabdomyosarcoma. Oncogene. $2001 ; 20: 5736--46$.

37. Xu M, Chen X, Chen D, et al. FoxO1: a novel insight into its molecular mechanisms in the regulation of skeletal muscle differentiation and fiber type specification. Oncotarget 2017;8:10662--74. 


\section{Figures}

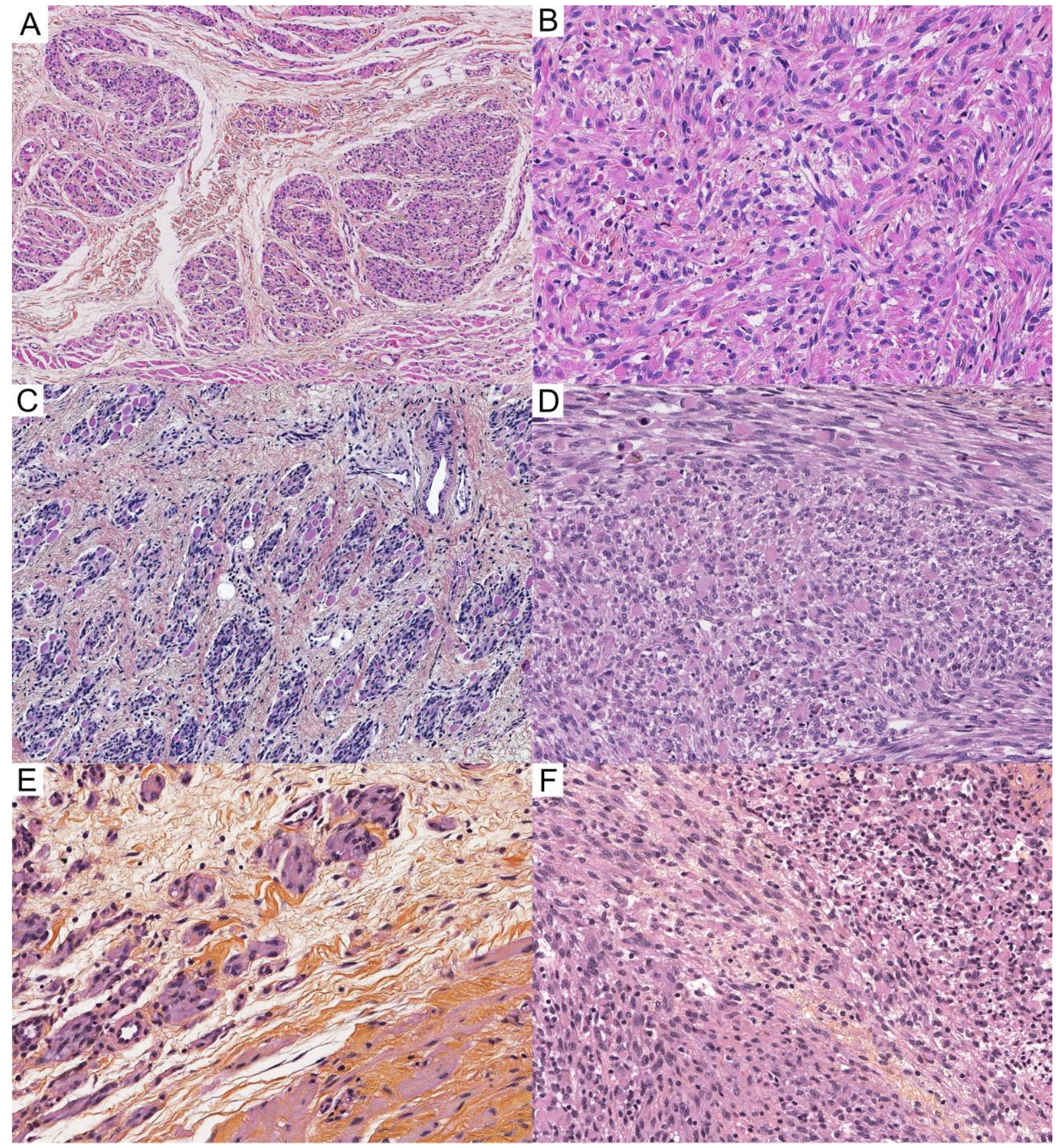

Figure 1: Histological features of the SRF-fused RMS (A, B case 1), (C, D case 2), (E, $F$ case 3). A, C, E, The periphery of the tumor consisted of small bundles in fibrous stroma (A, C HEx10, E HEx20). B, D, F, The central area was composed of long intersecting fascicles with a mixture of spindle and globoid cells. Tumor cells showed rhabdomyoblastic differentiation and nuclear atypia (HE x20). 

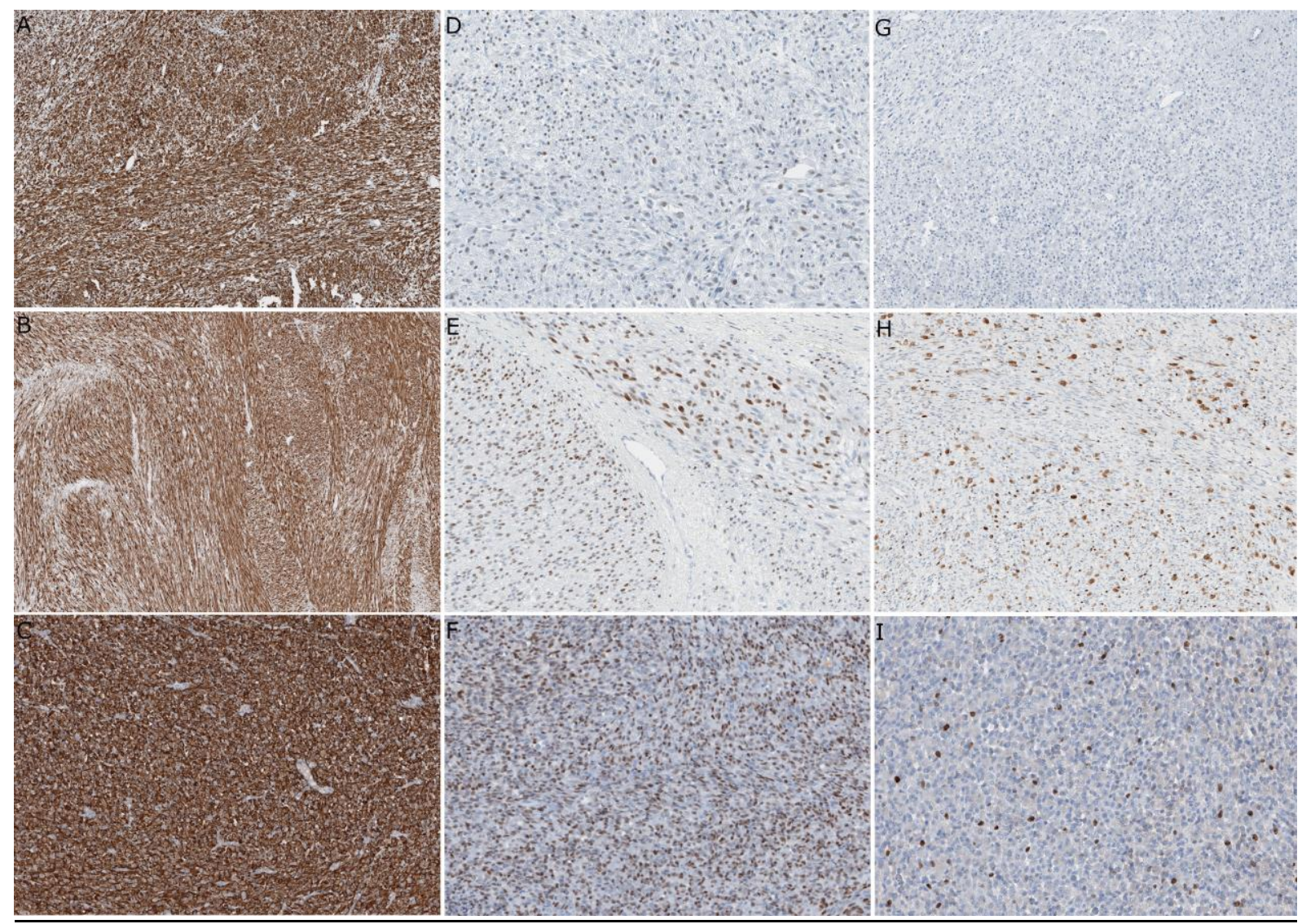

Figure 2: Immunohistochemical findings. Desmin was diffusely expressed (A case 1, B case 2, C case3). The staining for MyoD1 was heterogeneous (D case1, E case, $F$ case3). The Ki-67 staining was variable: $5 \%$ for case $1(\mathrm{G}), 20 \%$ for case $2(\mathrm{H}), 10 \%$ for case $3(\mathrm{I})$. 
A

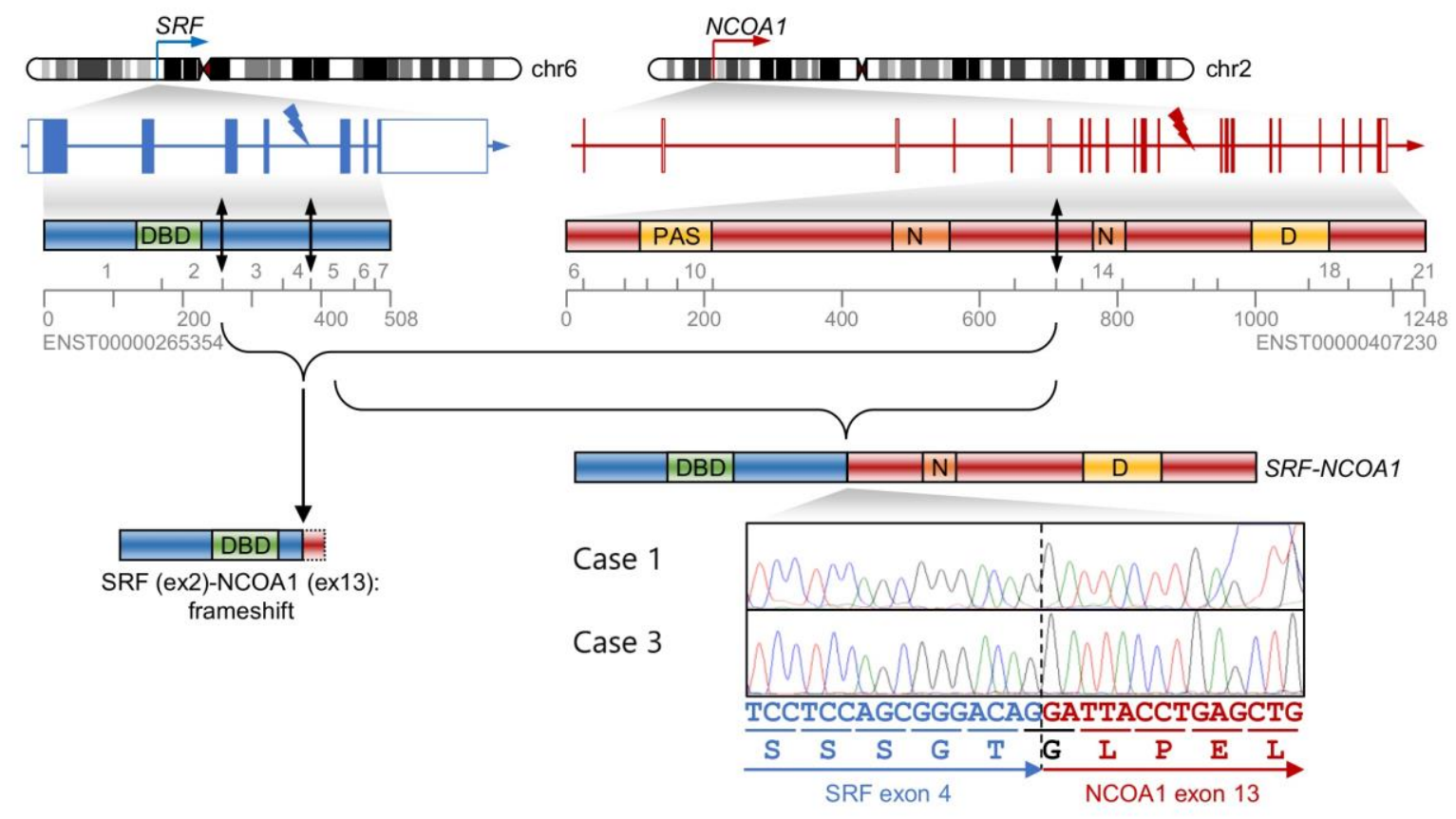

B

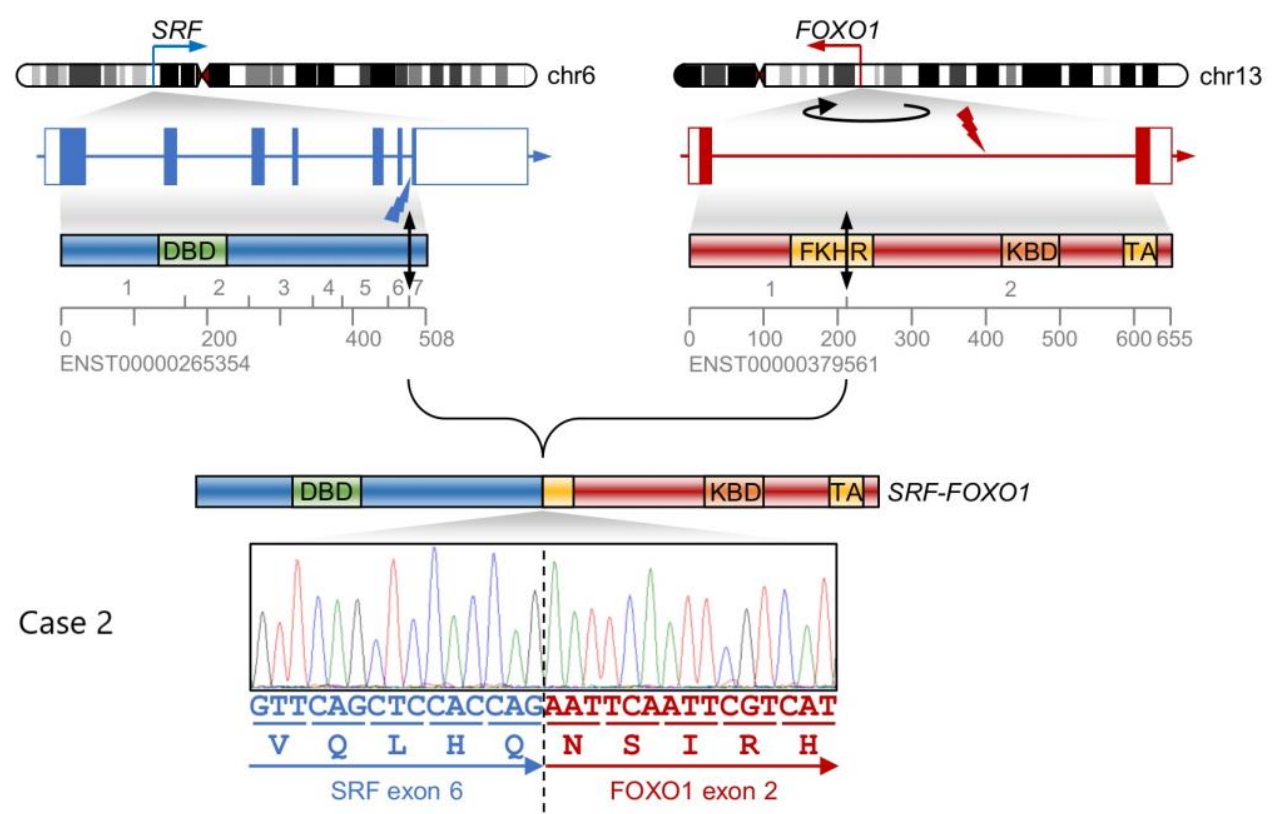

Figure 3: Schematic diagram of the fusion points and Sanger sequencing validation. A) SRF-NCOA1 fusions in case 1 and Case 3. Two different isoforms were present: the frameshift fusion of SRF exon 2 to NCOA1 exon 13 (left-hand diagram) and the inframe fusion of SRF exon 4 to NCOA1 exon 13 (right-hand diagram). Sanger sequencing, performed only for the in-frame fusions in both samples, is shown. B) SRF-FOXO1 fusion point in case 2. DBD: DNA binding domain, PAS: PAS-B protein interacting domain, $\mathrm{N}$ : Nuclear receptor coactivator, D: Domain of unknown function, FKHR: Forkhead DNA binding domain, KBD: KIX-binding domain (protein interacting domain), TA: Transcription activator domain. 


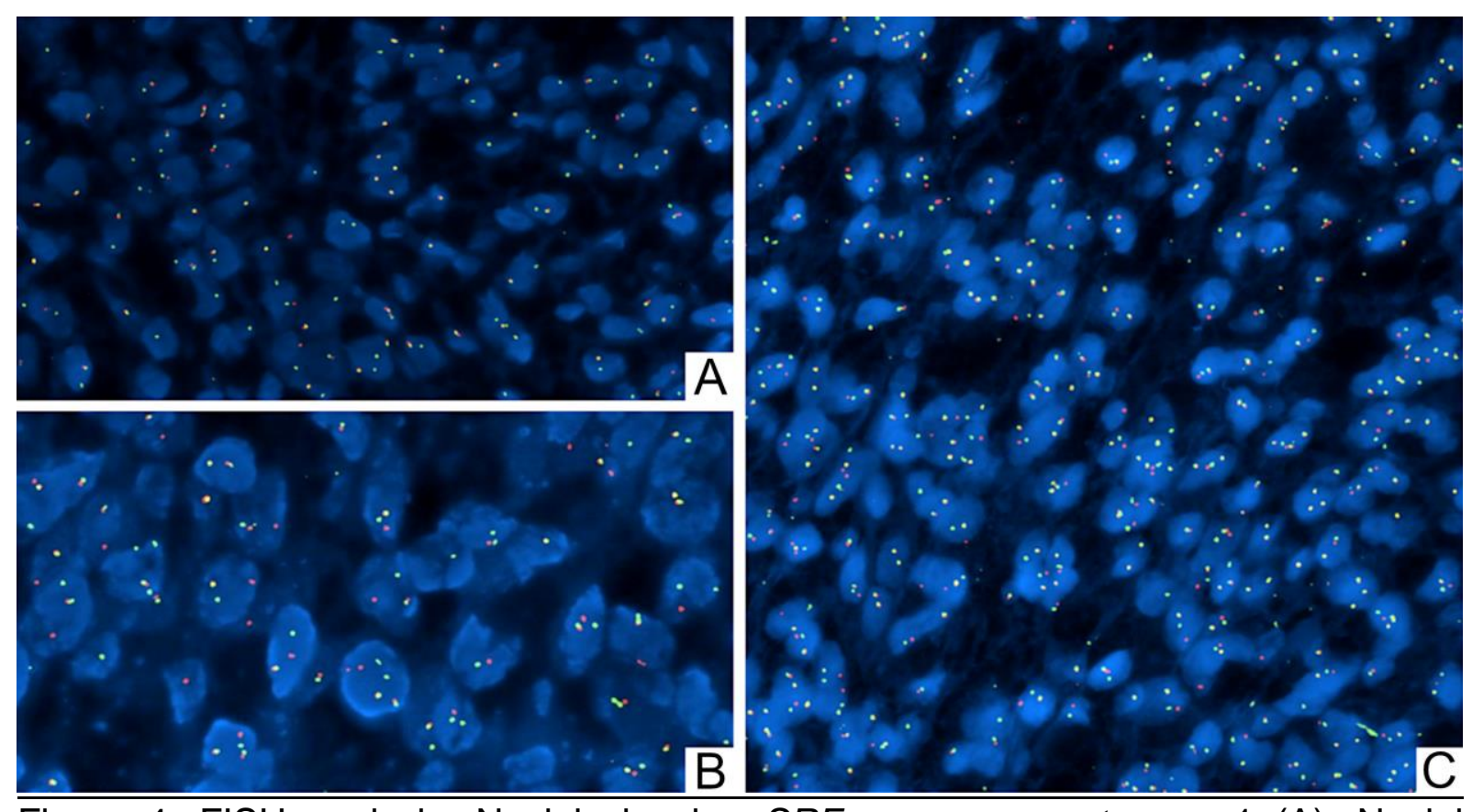

Figure 4: FISH analysis. Nuclei showing SRF rearrangement case $1(\mathrm{~A})$, Nuclei showing NCOA1 rearrangement in case $3(B)$, FOXO1 rearrangement in case 2 (C). 
A

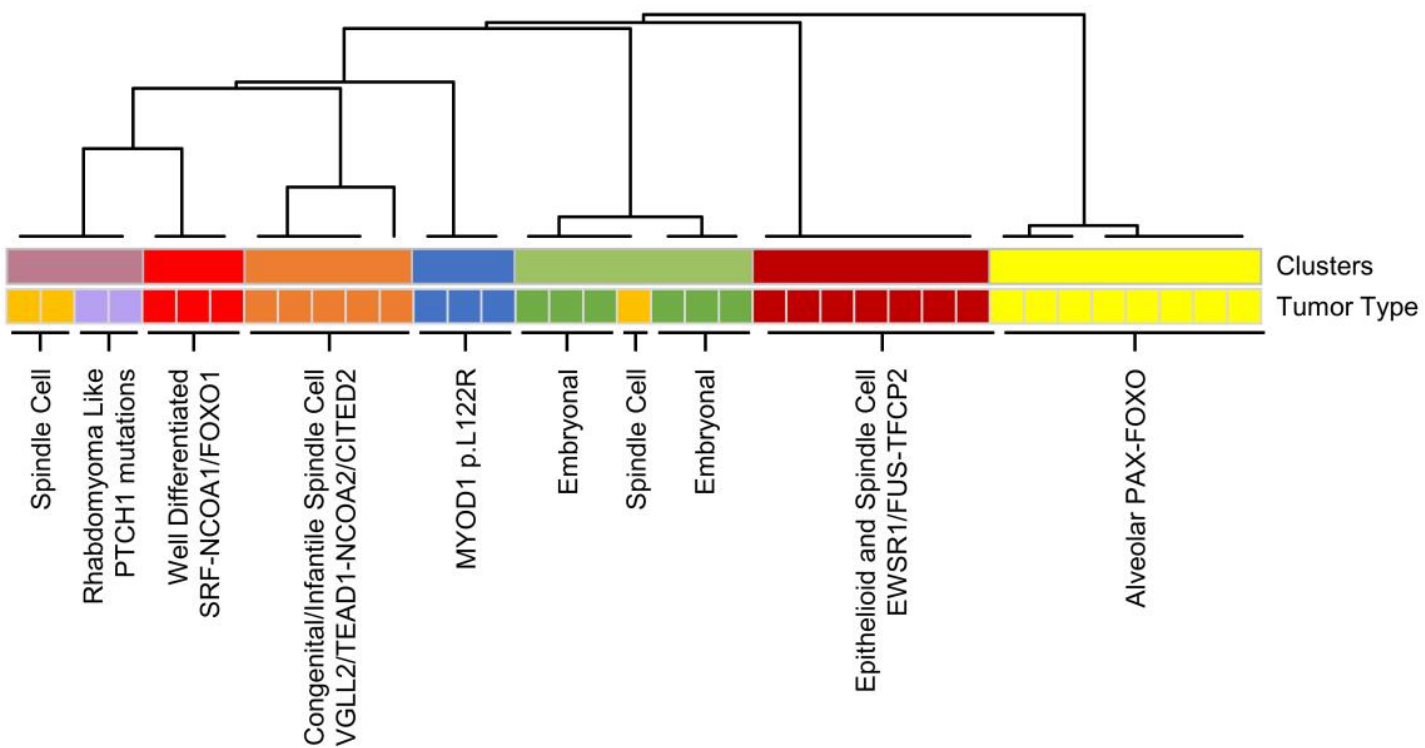

B

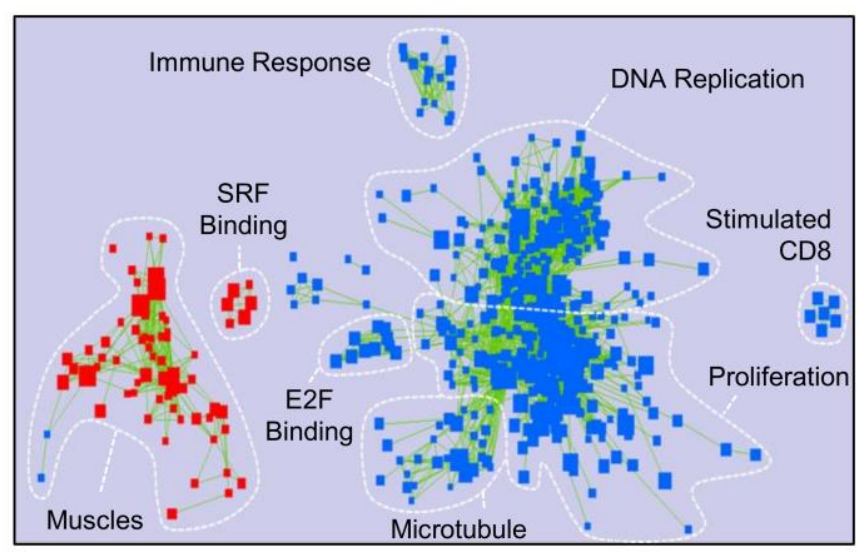

C

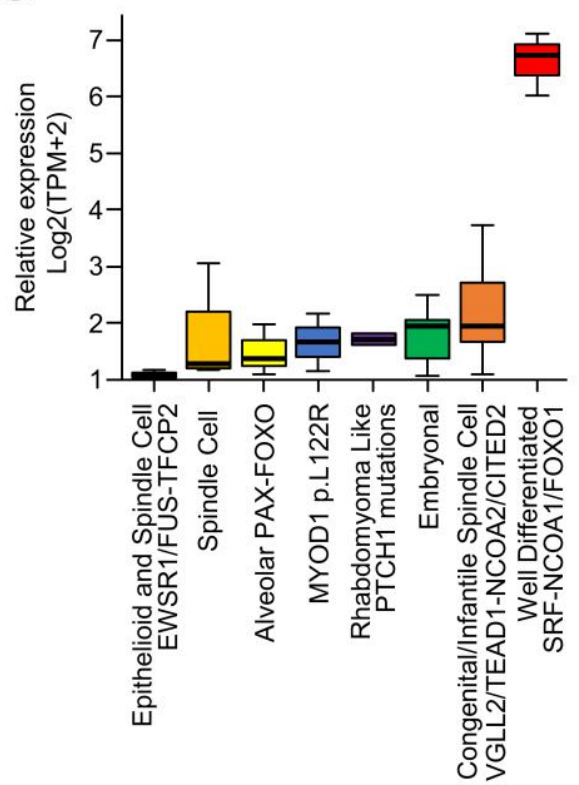

Figure 5: Specific expression signature assessed by RNA-sequencing. A) Unsupervised clustering analyses of skeletal muscle tumors. Consensus clustering was performed with 1000 permutations of $20 \%$ of the genes and $20 \%$ of the samples. B) Map of the significant GSEA gene sets either enriched (red) or depleted (blue) in $S R F$-fused RMSs compared to the other types of skeletal muscle tumors. C) SRFfused tumors present a high expression level of the ADAMTS8 transcript. TPM: Transcript per million. 


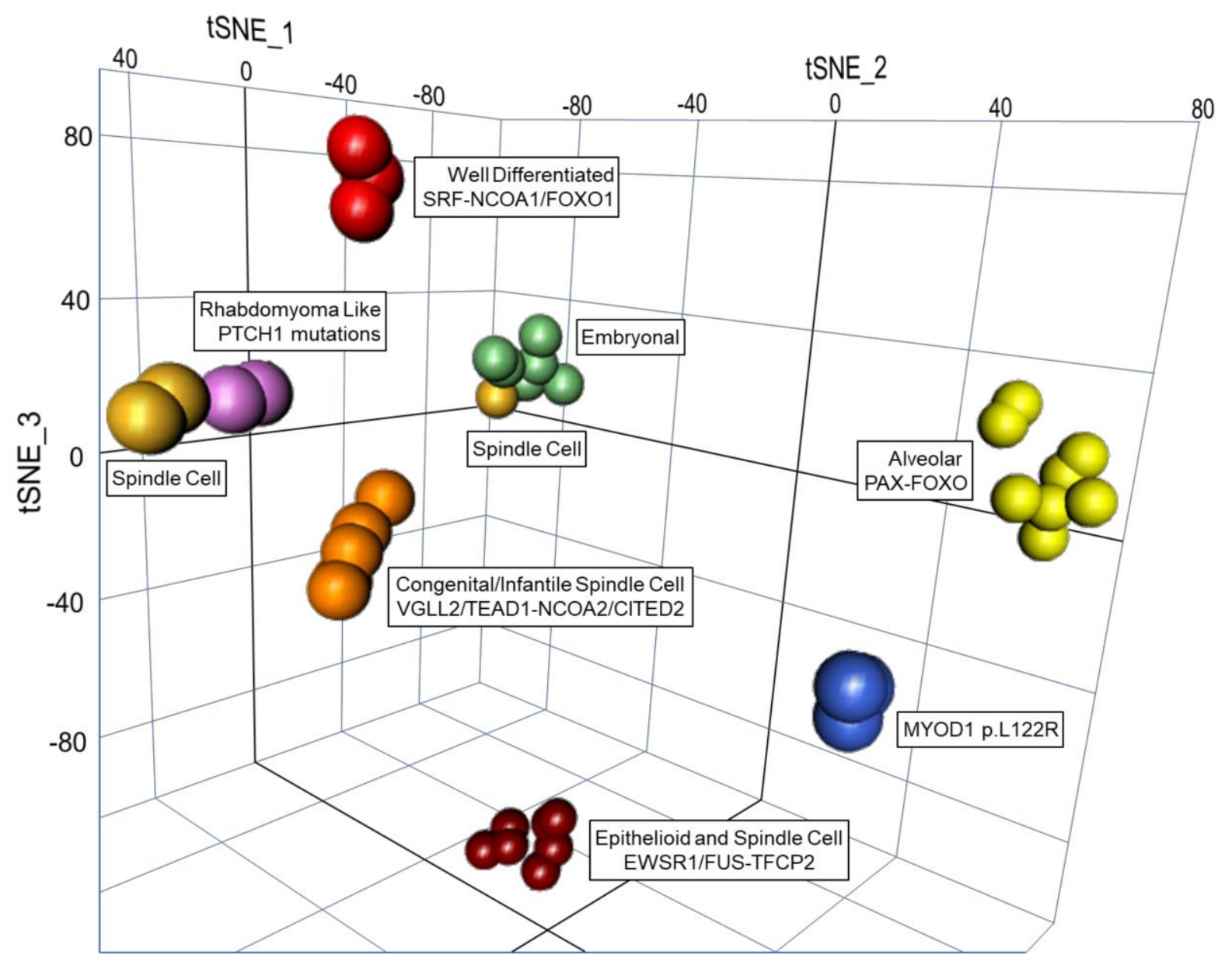

Figure 6: Expression data: t-SNE analysis. 


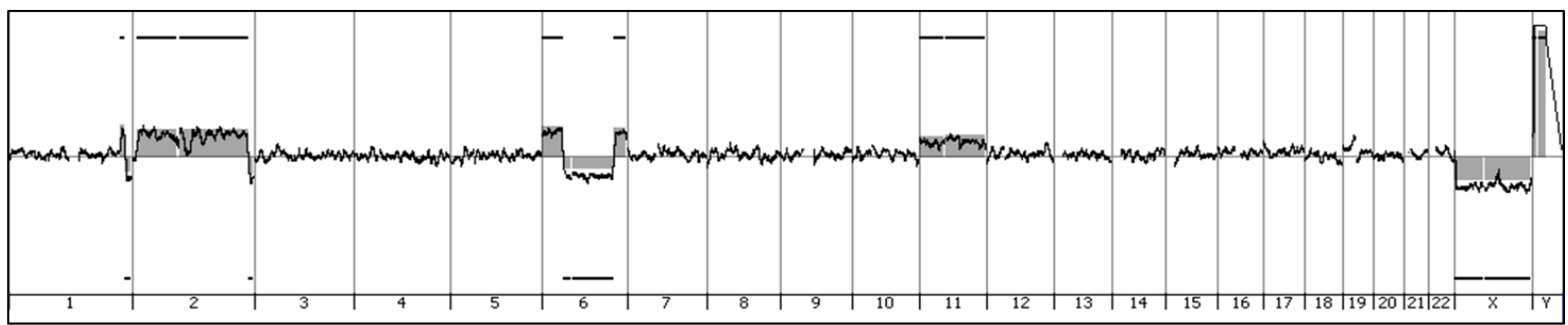

case_1

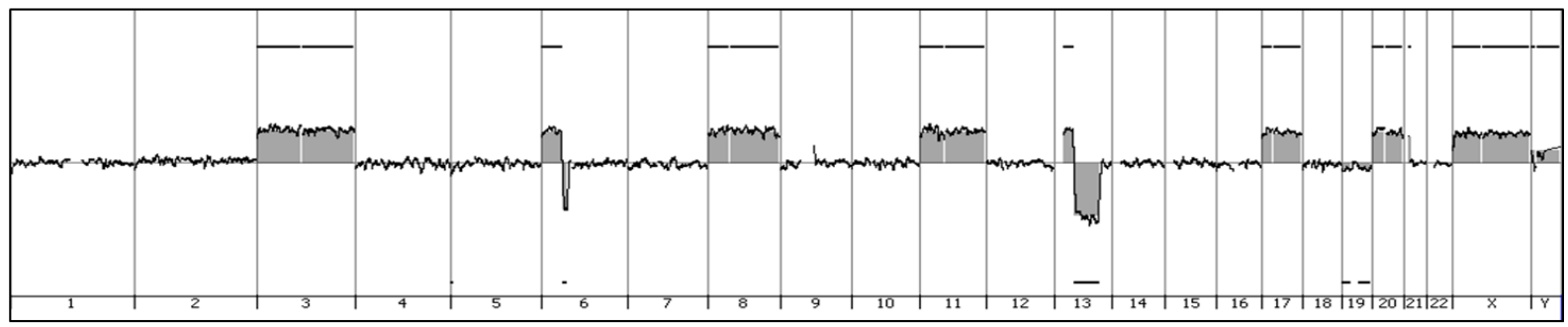

case_2

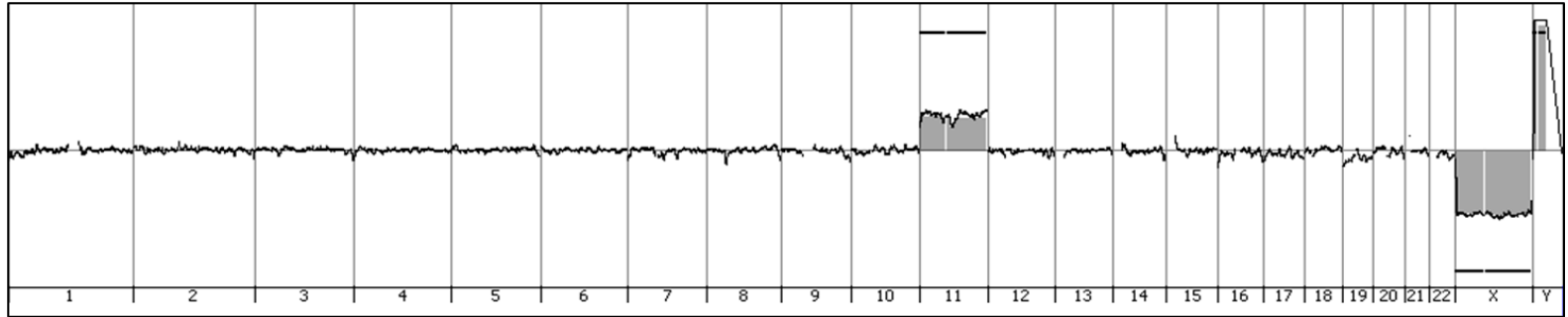

case_3

Figure S: Array comparative genomic hybridization showed a recurrent gain of the whole chromosome 11. 